\title{
Time-minimal set point transition for nonlinear SISO systems under different constraints
}

\author{
A. Himmel ${ }^{\text {a }}$, S. Sager ${ }^{\text {b }}$, K. Sundmacher ${ }^{\mathrm{a}, \mathrm{c}}$

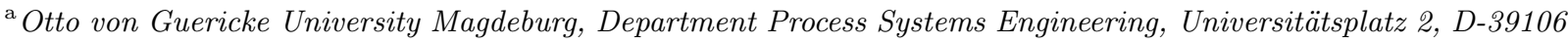 \\ Magdeburg, Germany

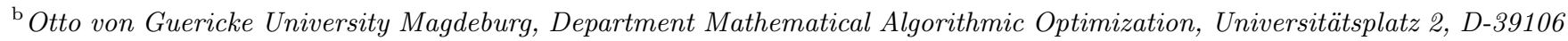 \\ Magdeburg, Germany \\ ${ }^{\mathrm{c}}$ Max Planck Institute for Dynamics of Complex Technical Systems, Department Process Systems Engineering, Sandtorstr.1, \\ D-39106 Magdeburg, Germany
}

\begin{abstract}
Set point transition of nonlinear plants plays an important role in many applications where dynamic process management has to be considered. This transition should be rapid - as operation in the new set point increases the economical benefit but in compliance with all safety regulations. We present a feedforward approach for a time-minimal set point transition of single-input, single-output nonlinear plants with respect to input, state and output constraints. The conceptual idea is based on the design of an optimization problem utilizing a coordinate change of the plant and a special setup function for the output trajectory. This allows the simultaneous planning of the trajectory and determination of the corresponding control signal. The formulation of the set point transition as an optimization problem provides a flexible design with respect to the integration of inequality constraints. Additional flexibility is provided by the type of setup functions, which permit any adjustment of the output trajectory between the set points. In contrast to other works, we focus on the stationarity of the system output, which allows a faster transition compared to the requirement to reach a steady state of all states. Finally, we present an example from the field of process systems engineering to demonstrate the applicability of the proposed methodology.
\end{abstract}

Key words: set point transition, optimization, nonlinear SISO systems

\section{Introduction}

This contribution considers the classical control engineering task of time-minimal set point transition. This entails the question of how to design a controller that brings a plant from one stationary set point $\hat{y}_{0}$ to a final value $\hat{y}_{T}$ as fast as possible. This type of problem occurs in many applications, such as robotics, aerospace or process systems engineering. An overview about different time-optimal state transition tasks can be found in the introduction of [5]. Frequently, the integration of different types of constraints during the transition process, to satisfy safety regulations or proper operation conditions, is of particular importance. Especially, if time-minimal set point transitions are required, bang-bang solutions should be avoided, as this can lead to increased wear of

Email addresses: himmel@mpi-magdeburg.mpg.de (A. Himmel), sager@ovgu.de (S. Sager), sundmacher@mpi-magdeburg.mpg.de (K. Sundmacher). equipment.

To illustrate our approach, we focus on a process systems engineering perspective of the set point transition scenario. We assume a hierarchical structure of the process operation management, where an upper control layer (e.g. a Real-Time Optimization Layer) specifies the new set points to a controller in a layer below, see [34,8,35]. Figure 1 illustrates this hierarchical control structure. Due to changing process conditions it might be efficient to adapt these set points to ensure an overall objective, e.g. profit maximization. The exact values for such set points depend on the specification of the operational objective, which is not discussed in detail in this contribution.

A suitable control structure has to generate a manipulating signal in a way, that the system moves from an initial output level to a final one. In this context, different constraints have to be satisfied to ensure a feasible and safe transition. 


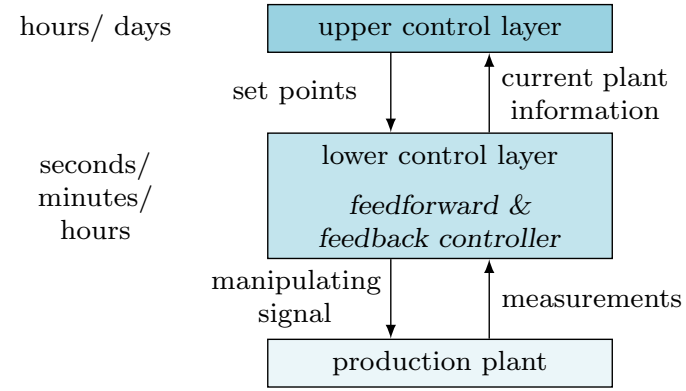

Fig. 1. Hierarchical control structure of a process and related time scales, typical for chemical process engineering.
Basically, one can distinguish between optimal output and state transition, see $[30,5,6]$. While the former refers to the plant output $y$ the later refers to the state coordinates which should move from an initial value $\hat{x}_{0}$ to a final value. Both problems are related to each other such that state transition techniques can be used to achieve a controlled output transition [5].

An additional way of classifying the transition apare used to determine the solution or not. For instance, the transition problem in [14] is explained by a twopoint boundary value problem. However, representatives of the first group are Model Predictive Controllers (MPC), where there exists many subtypes. The main idea of any MPC is that for a given reference signal, the MPC will compute a control signal by repeatedly solving an optimal control problem. In this context, the future plant behavior is predicted in every control step to ensure different kinds of constraints. For further information regarding MPC see $[26,15,27]$.

An important aspect is the reference signal, which is classically determined offline in advance. For the definition of the reference signal, it is useful to distinguish between trajectory tracking and path following, see [10].

The previously described controllers are usually applied online, i.e. in active operation. An alternative option are signal is determined offline in advance. Here, the concept of differential flatness is an important aspect which is 85 widely used in the literature for system transformation and determination of the input signal based on certain ansatz functions, see $[29,39,16]$. For a general introduction to this, the reader is referred to $[11,21]$. A wide variety of theoretical concepts exists for feedforward design to achieve a set point tracking, see e.g. [7,31,13]. ence trajectory defining the set point transition, see [36]. It can be represented by a time-dependent setup function. In the literature, various types of trajectory references can be distinguished. While in [14] or [32] polynomial or cosine-series are used as reference, in [12] Gevrey functions and in [37] splines are applied to avoid oscillations during set point changes. proaches depends on whether optimization techniques feedforward control strategies where the manipulation

One of the results of a feedforward control is the refer- 
Here $x \in \mathcal{X}, \operatorname{dim}(\mathcal{X})=n_{x}$ denotes the dynamical state, 51 $u \in \mathcal{U}, \operatorname{dim}(\mathcal{U})=1$ the manipulating variable and 52 $y \in \mathcal{Y}, \operatorname{dim}(\mathcal{Y})=1$ the plant output. We summarize the 53 right hand side of $(1 \mathrm{a})$ by $\tilde{X}(x, u):=\tilde{f}(x)+\tilde{g}(x) u$. It is assumed that there exist an unique state trajectory $x: \mathbb{R}^{+} \rightarrow \mathcal{X}$ as a solution of system $\tilde{\Sigma}$ for a given manipulating signal $u: \mathcal{X} \rightarrow \mathcal{U}$. In many applications in process systems engineering, the changing rate $\dot{u} \in \mathcal{U}_{d}:=\left\{\dot{u} \in \mathbb{R} \mid \underline{u}_{d} \leq \dot{u} \leq \bar{u}_{d}\right\}$ of the manipulating signal is bounded. Additionally, the following assumptions are valid.

Remark 1 Without loss of generality $\mathcal{X}$ can be repre- 60 sented locally by all values $x \in \mathbb{R}^{n_{x}}$ for those $\underline{x} \leq x \leq \bar{x}$. The same applies to $\mathcal{U}$ and $\mathcal{Y}$.

Assumption 2 For all output values $\hat{y} \in \mathcal{Y}$, there exists a manipulating value $\hat{u} \in \mathcal{U}$ and a state variable $\hat{x} \in$ $\hat{\mathcal{X}} \subseteq \mathcal{X}$ such that $0=\tilde{f}(\hat{x})+\tilde{g}(\hat{x}) \hat{u}, \hat{y}=h(\hat{x})$ and $\hat{x}$ is asymptotically stable.

The asymptotic stability is ensured by the use of a feedback controller.

As already mentioned, the objective is to find a control strategy that allows the plant $\tilde{\Sigma}$ to move between two stationary points. The time horizon of such a transition is denoted with $\tilde{\mathcal{T}}:=[0, T]$. A formal definition of the transitional task is given in Problem 5.

Definition 3 (Stationary set point) $A n$ output value $\hat{y}$ is called stationary at time $T$, if there exists an $s \in \mathbb{R}^{+}$, such that $\hat{y}=y(T)=y(T+s)$, when solving $\tilde{\Sigma}$ driven by a suitable manipulating signal $u(\cdot)$.

Remark 4 A conservative possibility to ensure stationarity of the set point imposes stationarity of all state coordinates, i.e. $\tilde{X}(\hat{x}, \hat{u})=0$ where $\hat{x} \mapsto h(\hat{x})=\hat{y}_{T}$. The corresponding state $\hat{x}$ is called steady state.

\section{Problem 5 (Time-minimal set point transition)}

Given the plant (1) with $\hat{y}_{0}=h\left(\hat{x}_{0}\right)$ and a new set point $\hat{y}_{T}$. Design a feedforward controller that generates a manipulating signal $u: \tilde{\mathcal{T}} \times \mathcal{X} \rightarrow \mathcal{U}$ that brings the system $\tilde{\Sigma}$ from an initial set point $\hat{y}_{0}=y(0)$ to a final stationary set point $\hat{y}_{T}=y(T)$. The set of all transition times $T$, for which there exists a signal $u: \tilde{\mathcal{T}} \times \mathcal{X} \rightarrow \mathcal{U}$ satisfying the system constraints, is denoted by $\mathbb{T}:=$ $\left\{T \in \mathbb{R}^{+} \mid 0<T \leq \infty\right\}$. A manipulating signal $u(t, x)$ is called suitable, if its domain is given by $\left[0, T^{*}\right] \times \mathcal{X}$ with $T^{*}=\inf \mathbb{T}$.

It should be noted that there are no terminal constraints 84 for the state variables $x$. Only the output variable $y$ is 85 fixed at the end of the transition horizon.

In the next section we give a brief introduction to some 87 theoretical basics which allow to consider the require- 88 ment of a stationary output signal in the solution of 89

the Problem 5. In addition, a method of nonlinear control is presented, which represents the basis for the optimization-based approach presented in Section 4. This inversion-based method uses a coordinate transformation to include input and output constraints. In Section 4 some modifications are made that allow a transformation into an optimization problem, where the transition time $T$ is explicitly minimized.

In order to calculate a manipulating signal $u(t, x)$ and the state trajectory from a given output signal, a local diffeomorphism $\Phi: \mathbb{R}^{n_{x}} \rightarrow \mathbb{R}^{n_{x}}$ is applied to change the coordinates. The first $r$ coordinates are given by

$$
\tilde{z}^{i}=\Phi^{i}(x):=\left(\mathrm{L}_{\tilde{f}}^{i-1} h\right)(x), \quad i \in \mathbb{I}
$$

whereas the last $n_{x}-r$ coordinates

$$
\tilde{z}^{r+j}=\Phi^{r+j}(x), \quad j \in \mathbb{J} .
$$

are not specified. We summarize the indices by the sets $\mathbb{I}:=\{1, \ldots, r\}$ and $\mathbb{J}:=\left\{1, \ldots, n_{x}-r\right\}$. The resulting system is called input-output normal form of $\tilde{\Sigma}$ and the last $n_{x}-r$ coordinates are called internal states. The unspecified functions in (2b) can be chosen arbitrarily if $\Phi$ is a diffeomorphism. 
Recall that one goal of Problem 5 is to determine a manipulating signal $u(t, x)$. Using (3) and (2), the component of the vector field of $\tilde{z}^{r}$ is influenced by the input $u$ and one can deduce

$$
\begin{aligned}
\dot{\tilde{z}}^{r} & =\left(\mathrm{L}_{\tilde{f}}^{r} h \circ \Phi^{-1}\right)(\tilde{z})+u\left(\mathrm{~L}_{\tilde{g}} \mathrm{~L}_{\tilde{f}}^{r-1} h \circ \Phi^{-1}\right)(\tilde{z}), \\
& =: \tilde{\alpha}^{-1}(\tilde{z}, u)
\end{aligned}
$$

and we obtain

$$
u=\frac{\dot{\tilde{z}}^{r}-\left(\mathrm{L}_{\tilde{f}}^{r} h \circ \Phi^{-1}\right)(\tilde{z})}{\left(\mathrm{L}_{\tilde{g}} \mathrm{~L}_{\tilde{f}}^{r-1} h \circ \Phi^{-1}\right)(\tilde{z})}=: \tilde{\alpha}_{z}\left(\tilde{z}, \dot{\tilde{z}}^{r}\right),
$$

or without using $\Phi^{-1}$

$$
u=\frac{\dot{\tilde{z}}^{r}-\left(\mathrm{L}_{\tilde{f}}^{r} h\right)(x)}{\left(\mathrm{L}_{\tilde{g}} \mathrm{~L}_{\tilde{f}}^{r-1} h\right)(x)}=: \tilde{\alpha}_{x}\left(x, \dot{\tilde{z}}^{r}\right) .
$$

First, we show how the constraints of the output, including its time derivatives, are integrated into this approach. These constraints are formulated as box constraints $y_{i} \leq y^{(i)} \leq \bar{y}_{i}, i \in \mathbb{I} \cup\{0\}$. Starting from a system (3) in normal input-output form, it is proposed to consider this type of constraints by applying another coordinate transformation $\Psi^{-1}: \tilde{z} \mapsto \xi$. This diffeomorphism maps the time derivative of the output to coordinates that are defined over the set of real numbers. The commutative diagram in Figure 2 illustrates the change of coordinates. This local diffeomorphism is given by

$$
\tilde{z}^{i}=\Psi^{i}(\xi)=\mu^{i}+\nu^{i} \psi^{i}\left(\xi^{i} ; \underline{\psi}_{i}, \bar{\psi}_{i}\right), i \in \mathbb{I}
$$

for the first $r$ coordinates and

$$
\tilde{z}^{r+j}=\Psi^{r+j}(\xi)=\xi^{r+j}, \quad j \in \mathbb{J}
$$

for the last $n_{x}-r$ coordinates. Here, $\mu^{i}, \nu^{i}, \psi_{i}$ and $\bar{\psi}_{i}$ are maps from the coordinates $\xi^{1}, \ldots, \xi^{i-1}$ to $\mathbb{R}$. However, for $i=1$ the corresponding functions are independent from $\xi$. The detailed strategy to calculate these maps can be deduced from the fact that $\tilde{z}^{i}$ is the time derivative of $\tilde{z}$. At this point, one can summarize that $\mu^{i}$ and $\nu^{i}$ result from applying the chain and product rule of differentiation. Hence, the terms contain an increasing number of partial derivatives. Indeed, these nonlinear terms can be determined using computer-algebra-systems like CasADi [1]. 
Remark 10 The first state coordinate is given by $\tilde{z}^{1}=47$ $\psi^{1}\left(\xi^{1} ; \underline{\psi}_{1}, \bar{\psi}_{1}\right)$. In this case $\mu^{1}=0, \nu^{1}=1, \underline{\psi}_{1}=\underline{y}_{0}{ }^{48}$ and $\bar{\psi}_{1}=\bar{y}_{0}$. The second coordinate can written as $\tilde{z}^{2}=$ $\frac{\partial \psi^{1}}{\partial \xi^{1}} \psi^{2}\left(\xi^{2} ; \underline{\psi}_{2}, \bar{\psi}_{2}\right)$. Again, $\mu^{2}=0$ but $\nu^{2}=\frac{\partial \psi^{1}}{\partial \xi^{1}}$ and the bounds are $\psi_{2}=y_{1}\left[\frac{\partial \psi^{1}}{\partial \xi^{1}}\right]^{-1}$ and $\bar{\psi}_{2}=\bar{y}_{1}\left[\frac{\partial \psi^{1}}{\partial \xi^{1}}\right]^{-1}$. bounds are $\psi_{2}=y_{1}\left[\frac{\partial \psi_{1}}{\partial 1}\right]$ and

The smooth saturation functions $\psi^{i}: \mathbb{R} \rightarrow\left[\underline{\psi}_{i}\left(\underline{y}_{i-1}\right)\right.$, $\left.\bar{\psi}_{i}\left(\bar{y}_{i-1}\right)\right]$ ensure the compliance with the output con- 55 straints. Therefore, the lower and the upper bound depend on the original output constraints, see [13] for details.

The transformation $\Psi$ only affects the first $r$ coordinates. The internal states remain constant, which implies an identity map for the last $n_{x}-r$ coordinates within $\Psi$. It follows from this local diffeomorphism that the resulting system has a triangular structure.

After all, the dynamic equations in these new coordinates are given by

$$
\Xi\left\{\begin{aligned}
\dot{\xi}^{i} & =\psi^{i+1}\left(\xi ; \underline{\psi}_{i+1}, \bar{\psi}_{i+1}\right), \quad i \in \mathbb{I} \backslash\{r\} \\
\dot{\xi}^{r} & =\psi^{r+1}\left(v ; \underline{\psi}_{r+1}, \bar{\psi}_{r+1}\right), \\
\dot{\xi}^{r+j} & =\mathrm{L}_{f} \Phi^{r+j} \circ \Gamma^{-1} \circ \Psi(\xi), j \in \mathbb{J}
\end{aligned}\right.
$$

where $v$ is a new input, that is given by a setup function $\Lambda(t ; p)$ satisfying the condition in Remark 7 automatically. The parameters $p$ are calculated by a numerical solver in oder to meet the $2 n_{x}$ initial and terminal constraints. Therefore, the number of parameters depends on the dimension of the system to avoid an overdetermined problem.

It should be noted that the vector field is generated by the saturation functions. For the interested reader, the detailed derivation of the system equation can be found in Appendix C. The compliance of the constraints for the first $r$ states $\tilde{z}$ or the output for the system $\tilde{\Sigma}$ is naturally included in the dynamic equation in $\Xi$.

Finally, we come to the input constraints. The conceptual idea is to map these constraints into the component of the vector field of $\tilde{z}^{r}$.

By inserting the lower and upper bounds of the input in (4) one can define $\underline{\tilde{\alpha}}_{z}^{-1}(\tilde{z}):=\min \left(\tilde{\alpha}_{z}^{-1}(\tilde{z}, \underline{u}), \tilde{\alpha}_{z}^{-1}(\tilde{z}, \bar{u})\right)$ and $\overline{\tilde{\alpha}}_{z}^{-1}(\tilde{z}):=\max \left(\tilde{\alpha}_{z}^{-1}(\tilde{z}, \underline{u}), \tilde{\alpha}_{z}^{-1}(\tilde{z}, \bar{u})\right)$. Next, using (7a) for the first $r$ coordinates one obtains $\underline{\tilde{\alpha}}_{\xi}^{-1}(\xi):=\underline{\tilde{\alpha}}_{z}^{-1} \circ \Psi(\xi)$ and $\overline{\tilde{\alpha}}_{\xi}^{-1}(\xi):=\overline{\tilde{\alpha}}_{z}^{-1} \circ \Psi(\xi)$. This way, the lower and upper bounds for the $r$ th derivative of the output signal are replaced by $\underline{\tilde{\alpha}}_{\xi}^{-1}=: \underline{y}_{r}$ and $\overline{\tilde{\alpha}}_{\xi}^{-1}=: \bar{y}_{r}$. The obtained expressions directly influence $\underline{\psi}_{r+1}$ and $\bar{\psi}_{r+1}$ in $(8 \mathrm{~b})$.

$\overline{\mathrm{A}}^{r}$ further aspect, presented in [13], is a method to determine the transition time depending on the utilization of the input constraints. Therefore, a parameter $\delta \in(0,1)$ is introduced to measure the aggressiveness of the feedforward control. Specifying a certain value for $\delta$ means to get a corresponding transition time $T(\delta)$. For $\delta \rightarrow 1$, the input constraints are highly utilized and the manipulating signal becomes a bang-bang control. To this end, two additional ODEs (next to (8)) and three boundary conditions have to be included to adjust the transition time $T$ as an additional parameter.

\section{Time-Minimal Transition Problem}

In the previous section, we discussed a state of the art method to solve constrained transition problems. The disadvantage of this approach is the fact that only a transition between two stationary states is allowed. In addition, the method only allows the consideration of input and output constraints by a further coordinate transformation. A direct optimisation of the transitional period is also not possible, which is also reflected in the formulation of a BVP.

In this section we present a modification of the setup function that allows a formulation as optimization problem and thus also the integration of inequality constraints. Finally, we want to compare this approach with the one discussed in the previous section.

\subsection{System Formulation}

In the following the plant $\tilde{\Sigma}$ is time transformed via $t=\tau T$ in oder to map the time horizon $[0, T]$ of the transition to the fixed time horizon $[0,1]$. So, $T$ becomes a parameter of the system. This technique is widly use in the literature, see e.g. $[22,24]$. The plant dynamic (1a) is reformulated as follows $\dot{x}_{\tau}=T \tilde{f}(x)+T \tilde{g}(x) u$, where the subscript $\tau$ indicates a time derivative with respect to $\tau$. Defining $f(x):=T \tilde{f}(x)$ and $g(x):=T \tilde{g}(x)$ yields the modified plant model

$$
\Sigma\left\{\begin{aligned}
\dot{x}_{\tau} & =f(x)+g(x) u, x(0)=x_{0} \\
y & =h(x) .
\end{aligned}\right.
$$

The state and input constraints given by $\mathcal{X}$ and $\mathcal{U}$ remain unaffected. An exception is the constraint for the input derivative $\mathcal{U}_{d}$, whose lower and upper bounds are weighted by the transition parameter $T$.

Remark 11 For the sake of readability, we dispense with the explicit mention of the subscript ${ }_{\tau}$.

In equivalence to the previous section, we apply the coordinate transformation (2), where the vector field $X(x, u)=f(x)+g(x) u$ is used. The new state coordinate is denoted by $z$, where its components are given by

$$
z^{i}=\Gamma^{i}(x):=\left(\mathrm{L}_{f}^{i-1} h\right)(x), \quad i \in \mathbb{I}
$$


and $z^{j}=\Gamma^{j}(x)=\Phi^{j}(x), j \in \mathbb{J}$. A relation between $z$ and $\tilde{z}$ is given in Lemma 12 .

Lemma 12 (Coordinate transformation $z \mapsto \tilde{z}$ ) Consider the transformation law (2) related to the vector field $\tilde{X}$. The coordinates of the non-time transformed system are given by

$$
\begin{aligned}
\tilde{z}^{i} & =T^{-(i-1)} z^{i}, & & i \in \mathbb{I} \\
\tilde{z}^{r+j} & =z^{r+j}, & & j \in \mathbb{J} .
\end{aligned}
$$

The proof can be found in Appendix A. In the following we proceed from the time-transformed plant $\Sigma$ in inputoutput normal form given by (12). Here, the explicit inversion of the local diffeomorphism $\Gamma$ is omitted. This makes it easier to generate the optimization problem automatically later. For this purpose, the state transformation is seen as an additional algebraic equation.

$$
\begin{aligned}
\dot{z}^{i} & =\left(\mathrm{L}_{X} \Gamma^{i}\right)(x), \quad i \in \mathbb{I}_{\mathcal{X}} \\
0 & =\Gamma(x)-z .
\end{aligned}
$$

As introduced in the previous section, we consider a suitable time and parameter dependent setup function $\Lambda_{t_{f}}:\left[0, t_{f}\right] \times \mathbb{R}^{r+1} \times \mathbb{R}^{n_{q}} \rightarrow \mathcal{Y},(t, p, q) \mapsto \Lambda_{t_{f}}(t, p, q)$. This function is at least $r+1$ times continuously differentiable and describes the time evolution of the output $y$ to realize the time-minimal set point transition as introduced in Problem 5. As we are looking at a timetransformed plant here, let $t_{f}=1$. There are two types of parameters in $\Lambda_{t_{f}}$. The first one is indicated with $p \in \mathbb{R}^{r+1}$ and its number is directly connected to the relative degree of the system. The number of the sec- 23 ond parameter group $q \in \mathbb{R}^{n_{q}}$ can be chosen arbitrarily due to two reasons: in our case we have no terminal constraints which fix the state $x$ at $\tau=1$ and due to the fact that we formulate the transition problem as an optimization problem we can handle underdetermined systems. Indeed, the set point transition can be comprehended as a parameter estimation problem, where the objective to be minimized is the transition time $T$.

By integrating the setup function $\Lambda_{1}$ into the plant equations, the number of dynamic equations can be reduced, since the trajectories $\left(z^{1}, \ldots, z^{r}\right)$ are defined in advance. Thus the first $r$ equations of the diffeomorphism can be written as

$$
0=\left(\mathrm{L}_{f}^{i-1} h\right)(x)-\Lambda_{1}^{(i-1)}(\tau ; p, q), i \in \mathbb{I} .
$$

The remaining equations of the transformation are

$$
0=\Gamma^{r+j}(x)-z^{r+j}, j \in \mathbb{J}
$$

where the time evolution of the states is to be determined by the solution of the ODE

$$
\dot{z}^{r+j}=\left(\mathrm{L}_{X} \Gamma^{r+j}\right)(x), \quad j \in \mathbb{J} .
$$

4 In summary, the inverted system is given as follows

$$
\Upsilon\left\{\begin{array}{rlrl}
\dot{z}^{r+j} & =\left(\mathrm{L}_{X} \Gamma^{r+j}\right)(x), & j \in \mathbb{J} \\
0 & =\left(\mathrm{L}_{f}^{i-1} h\right)(x)-\Lambda_{1}^{(i-1)}(\tau ; p, q), & i \in \mathbb{I}, \\
0 & =\Gamma^{r+j}(x)-z^{r+j}, & & j \in \mathbb{J} . \\
0 & =\alpha_{x}\left(x, \Lambda_{1}^{(r)}(\tau ; p, q)\right)-u & &
\end{array}\right.
$$

Remark 13 In (13d) we refer to $X$ and thus renounce the $\sim$ above the $\alpha$.

A reason for using (13d) is that the dynamics of the internal states can be dependent on the input $u$. However, if $\mathrm{L}_{g} \Gamma^{r+j}(x)=0, j \in \mathbb{J}$ is fulfilled, then $(13 \mathrm{~d})$ can be omitted when solving $\Upsilon$. The lifted system $\Upsilon$ includes $2 n_{x}-r+1$ equations with $n_{x}-r$ ODEs and $n_{x}+1$ algebraic equations. In this context, the variables $x$ and $u$ have algebraic nature.

A significant reduction of $\Upsilon$ can be achieved if the internal states $z^{r+i}, i \in \mathbb{J}$ are choosen suitable. For instance, if a subset of the original state coordinates is used, (13c) does not have to be considered. For this we define $\Gamma^{r+j}(x):=x^{m_{j}}$ where $m_{j} \in \mathbb{I}_{\mathcal{X}, \mathrm{r}}:=$ $\left\{k \in \mathbb{I}_{\mathcal{X}}\right\},\left|\mathbb{I}_{\mathcal{X}, \mathrm{r}}\right|=n_{x}-r$ and $j \in \mathbb{J}$. Note that this is only possible if the map $\Gamma$ generated in this way is a diffeomorphism. For the reduced system we obtain

$$
\Upsilon_{\mathrm{r}}\left\{\begin{array}{rlrl}
\dot{x}^{m_{j}} & =f^{m_{j}}(x)+g^{m_{j}}(x) u, & & j \in \mathbb{J} \\
0 & =\left(\mathrm{L}_{f}^{i-1} h\right)(x)-\Lambda_{1}^{(i-1)}(\tau ; p, q), & i \in \mathbb{I}, \\
0 & =\alpha_{x}\left(x, \Lambda_{1}^{(r)}(\tau ; p, q)\right)-u &
\end{array}\right.
$$

which is composed of $n_{x}+1$ equations.

\subsection{Formulation of the Optimization Problem}

So far we have generated the inverted system (13) and (14), respectively. In order to formulate an optimization problem for deriving the minimum transition time $T^{*}$ and the associated manipulation signal $u^{*}:\left[0, T^{*}\right] \times \mathcal{X} \rightarrow \mathcal{U}$, we have to address some issues.

As already mentioned, the time derivative of the input $u$ plays an important role in the set point transition in various applications. To get an expression for it, we differentiate a time transformed version of (4) one more time with respect to $\tau$. Rewriting the derived equation we obtain

$$
\begin{aligned}
& \dot{u}= \frac{\Lambda_{1}^{(r+1)}(\tau ; p, q)-\left(\mathrm{L}_{X} \mathrm{~L}_{f}^{r} h\right)(x)}{\left(\mathrm{L}_{g} \mathrm{~L}_{f}^{r-1} h\right)(x)} \\
&+\frac{u\left(\mathrm{~L}_{X} \mathrm{~L}_{g} \mathrm{~L}_{f}^{r-1} h\right)(x)}{\left(\mathrm{L}_{g} \mathrm{~L}_{f}^{r-1} h\right)(x)} \\
&=\gamma(\tau, x, u ; p, q) .
\end{aligned}
$$


In order to formulate the optimization problem, we discretize the time horizon $\mathcal{T}$ by $N$ subintervals. The sampling period $\Delta \tau_{k}$ of each subinterval can be chosen arbitrarily but must satisfy the condition $\sum_{k=1}^{N} \Delta \tau_{k}=1$. We further define

$$
\mathbb{S}:=\left\{\tau_{k} \in \mathbb{R}^{+} \mid \tau_{k}=\sum_{i=1}^{k} \Delta \tau_{k}, k=1, \ldots, N\right\}
$$

Furthermore, the initial and boundary conditions have 30 o be determined. From (2) and (6) we obtain $z_{0}:=31$ ) as initial condition for the dynamic states and 32 $a_{x}\left(x_{0}, 0\right)$ for the input variable. This The boundaries for the input variable remain untouched by the reformulation of the system. Considering (15), the derivative $\dot{u}$ is with respect to $\tau$. Thus, for the set of admissible values we obtain $\underline{u}_{d} \leq T^{-1} \gamma(\tau, x, u ; p, q) \leq \bar{u}_{d}$.

The solution of the plant model $\Upsilon$ at time $\tau$ is described by a one step integration through $\phi_{\tau}\left(w_{0} ; q\right):=$ $(x(\tau), z(\tau), u(\tau))$, where $w_{0}:=\left(x_{0}, z_{0}, u_{0}\right)$ summarizes the initial values. Here we assumed that the relative degree $r$ is known, which means that the parameters $p$ are given. Finally, we are able to formulate the optimization problem as an NLP of the form

$$
\begin{array}{ll}
\underset{T, q}{\operatorname{minimize}} T & \\
\text { subject to } & \\
(\underline{x}, \underline{z}, \underline{u}) \leq \phi_{\tau_{k}}\left(w_{0} ; q\right) \leq(\bar{x}, \bar{z}, \bar{u}) & \tau_{k} \in \mathbb{S}, \\
\underline{u_{d}} \leq T^{-1} \gamma\left(\tau_{k}, x\left(\tau_{k}\right), u\left(\tau_{k}\right) ; p, q\right) \leq \overline{u_{d}} & \tau_{k} \in \mathbb{S} .
\end{array}
$$

Assumption 14 It is assumed that for suitable boundaries the optimization problem (16) is feasible.

Remark 15 The definition of $\mathbb{S}$ and thus the step sizes $\Delta \tau_{k}$ of the time grid is affected by two aspects. The first aspect is related to the implemented solution technique. For instance, using a direct single/multiple shooting one might apply an equidistant grid. If an orthogonal collocation is used, $\Delta \tau_{k}$ is determined by the zeros of orthogonal Legendre polynomials. A further aspect concerns the accuracy in the evaluation of the trajectories and thus the constraints. However, the focus of this work is not on the comparison of different discretisation methods, hence we refer to the corresponding literature [23,22,3,4].

Remark 16 Problem 16 has a local solution if there exist a state $x$ in the preimage of the new set point $\hat{y}_{T}$ that is reachable from $\hat{y}_{0}$, see [28,18].

As discussed in Section 3.1, the approach in [14] satisfies output constraints. If these types of constraints occur in addition to the state constraints, two things have to be noted. First, the lower and upper boundaries have to be mapped to the $z$ coordinates using Lemma 12. Second, the boundaries thus created may differ from those resulting from diffeomorphism. In this case, the more restrictive result for $\underline{z}$ or $\bar{z}$ has to be used.

\subsection{Setup Function}

In this subsection, the setup function $\Lambda_{1}$ that is essential to solve the transition problem (16) is discussed in more detail. As we could see above, the function $\Lambda_{1}$ plays a major role in the system $\Upsilon$, since it specifies the time evolution of the output $y$ and its time derivatives. Considering the objective of the transition problem, one can describe the set of admissible setup function as follows.

Definition 17 (Admissible Set Up Function ) $A$ setup function $\Lambda_{t_{f}}:\left[0, t_{f}\right] \times \mathbb{R}^{r+1} \times \mathbb{R}^{n_{q}} \rightarrow \mathcal{Y}$ given by

$$
\Lambda_{t_{f}}(t ; p, q):=\hat{y}_{0}+y_{\Delta} \lambda_{t_{f}}(t ; p, q) \text {. }
$$

where $y_{\Delta}:=\hat{y}_{T}-\hat{y}_{0}$, is called an admissible candidate for Problem 5, if $\lambda_{t_{f}}(t ; p, q)$ satisfies

$$
\begin{aligned}
& \text { (i) } 0=\lambda_{t_{f}}^{(k)}(0 ; p, q), \quad k=0, \ldots, r, \\
& \text { (ii) } 1=\lambda_{t_{f}}\left(t_{f} ; p, q\right), \\
& \text { (iii) } 0=\lambda_{t_{f}}^{(k)}\left(t_{f} ; p, q\right), \quad k=1, \ldots, r .
\end{aligned}
$$

We propose that $\lambda_{t_{f}}(t ; p, q)$ is a summation of two terms in the sense of

$$
\lambda_{t_{f}}(t ; p, q)=A_{t_{f}}(t ; p)+B_{t_{f}}(1 ; q)
$$

where $A_{t_{f}}$ is called the basic and $B_{t_{f}}$ variation term. The basic term fulfills the boundary conditions of Definition 17 and depends only on the relative degree $r$ of the system $\Sigma$. The parameters $\left\{p_{i}\right\}_{i=1, \ldots, r+1}$ are fix, and they shall ensure that there exists a trajectory between $\hat{y}_{0}$ and $\hat{y}_{T}$.

In contrast, the variation term $B_{t_{f}}$ serves to adapt the trajectory within the time horizon $\left[0, t_{f}\right]$, whereas on the boundaries $B_{t_{f}}^{(k)}(0 ; q)=B_{t_{f}}^{(k)}\left(t_{f} ; q\right)=0, k=0, \ldots, r$. To this end, the parameters $\left\{q_{v}, i\right\}_{i=1, \ldots, N_{v}}$ are free and $N_{v}$ can be arbitrarily chosen. In other words, $N_{v}$ determines the degree of freedom for the transition problem.

Table 1

Ansatz functions for $\lambda_{t_{f}}$ where $\hat{\tau}:=t / t_{f}$.

\begin{tabular}{|l|l|l|}
\hline & Polynomial & Trigonometric \\
\hline$A_{t_{f}}(t ; p)$ & $\sum_{i=1}^{r+1} p_{i} \hat{\tau}^{r+i}$ & $\sum_{i=1}^{r+1} p_{i} \cos ((i-1) \pi \hat{\tau})$ \\
\hline$B_{t_{f}}(t ; q)$ & $\sum_{i=1}^{N_{v}} q_{i} \hat{\tau}^{i}\left(\hat{\tau}^{2}-\hat{\tau}\right)^{r+2}$ & $\sum_{i=1}^{N_{v}} q_{i} \sin ^{r+2}(i \pi \hat{\tau})$ \\
\hline
\end{tabular}




\subsection{Application of the Control Law}

The solution of the optimization problem (16) for a given setup function from Table 1 yields optimal parameters $q^{*}$ as well as a minimum transition time $T^{*}$. The result is used to design the feedback controller

$$
\begin{aligned}
K_{w_{0}}: \mathbb{R} \times \mathcal{X} & \rightarrow \mathcal{U}, \\
(t, x) & \mapsto K_{w_{0}}(t, x),
\end{aligned}
$$

for the plant $\tilde{\Sigma}$, where

$K_{w_{0}}(t, x):= \begin{cases}\tilde{\alpha}_{x}\left(x, \Lambda_{T^{*}}^{(r)}\left(t ; p, q^{*}\right)\right)+\beta(t, x) & t \in\left[0, T^{*}\right] \\ \sigma(x)+\beta\left(T^{*}, x\right) & t>T^{*}\end{cases}$

and

$$
\sigma(x):= \begin{cases}\tilde{\alpha}_{x}(x, 0) & \text { internal dynamic is stable } \\ \hat{u}_{T} & \text { internal dynamic is unstable }\end{cases}
$$

Here, $\tilde{\alpha}_{x}$ is responsible for the transition part and it can be interpreted as feedforward control. As common in literatur, $\beta$ is an additional feedback part of the control law. Even though $\tilde{\alpha}_{x}$ will bring the plant along the nominal trajectory to the desired set point $\hat{y}_{T}, \beta$ can be used to additionally stabilize the plant along this nominal trajectory despite uncertainties. This approach is known in literatur as "two-degree-of-freedom control" and has already been used for nonlinear systems, 61 see $[37,20]$. For instance, the map $\beta$ can be represented 62 by a PI controller or a LQR that is designed using a lin- 63 earization along the nominal state trajectory generated 64 by applying $\tilde{\alpha}_{x}$. Further information on the design can 65 be found in $[25,2,40]$ The subscript $w_{0}$ emphasizes that ${ }_{66}$ the controller was designed for a certain initial value of 67 $\tilde{\Sigma}$. Even if Assumption 2 requires that $\tilde{\Sigma}$ and thus the 68 transformed system $\Upsilon$ is stable, the pure application 69 of $\tilde{\alpha}_{x}$ can cause a destabilization. For this reason, we 70 have to discuss two scenarios below that differ for times 71 $t>T^{*}$ after the transition.

For stable internal dynamic, the control law during and 73 after the transition consits of both parts $\tilde{\alpha}_{x}$ and $\beta .74$ Due to Assumption 14, within $\left[0, T^{*}\right]$ the trajectories 75 are bounded via the constraints $(16 \mathrm{~b})$. The part $\tilde{\alpha}_{x}$ guarantees both the transition to the new set point $\hat{y}_{T} \quad 76$

\subsection{Final Comments}

In Section 3, we introduced the approach of [14]. This was applicable to systems with output and input constraints using a special diffeomorphism with saturation functions. A time-dependent setup function converts the transition problem into a BVP where $n_{x}$ parameters needs to be determined as the system moves from an initial steady state to a final one. Depending on a predefined factor $\delta$ to model the aggressiveness of the manipulating signal, the transition time $T$ becomes another parameter that has to be determined. This is associated with the presence of input constraints. Using gradientbased optimization techniques, to minimize the transition time directly, causes numerical difficulties due to discontinuity operations like $\min / \max$ operations, taking the absolute value or case dependent functions.

The significant difference to the method described before 


\section{Table 2}

Comparison of the classic inversion-based control design approach (IFD) and the proposed time-minimal set point transition problem (tmSTP) approach.

\begin{tabular}{|l|l|l|}
\hline & IFD & tmSTP \\
\hline System & $\Xi$ & $\Upsilon$ or $\Upsilon_{\mathrm{r}}$ \\
Time optimization & No & Yes \\
DOF & $n_{x}+1$ & $N_{v}+1 \geq 1$ \\
Constraints & $\mathcal{U}$ and $\mathcal{Y}$ & $\mathcal{U}_{d}, \mathcal{U}, \mathcal{X}$ and $\mathcal{Y}$ \\
Solution w.r.t. Prob. 5 & not suitable & suitable \\
\hline
\end{tabular}

\section{Example: van de Vusse reactor}

To demonstrate the proposed approach, we consider the control of a van de Vusse reactor [38] is a continuously stirred tank reactor (CSTR) [33]. Inside the reactor the inlet feed stream of component $\mathrm{A}$ is converted according to the reaction scheme $\mathrm{A} \longrightarrow \mathrm{B} \longrightarrow \mathrm{C}, 2 \mathrm{~A} \longrightarrow \mathrm{D}$. The dynamics of this production plant is given by

$$
\begin{aligned}
\dot{c}_{A} & =q\left(c_{A, i n}-c_{A}\right)-k_{1}(\vartheta) c_{A}-k_{2}(\vartheta) c_{A}{ }^{2} \\
\dot{c}_{B} & =-q c_{B}+k_{1}(\vartheta) c_{A}-k_{1}(\vartheta) c_{B} \\
\dot{\vartheta} & =q\left(\vartheta_{i n}-\vartheta\right)+\kappa_{1}\left(\vartheta_{c}-\vartheta\right)+h\left(c_{A}, c_{B}, \vartheta\right) \\
\dot{\vartheta}_{c} & =\kappa_{2}\left(\vartheta-\vartheta_{c}\right)+\kappa_{3} Q,
\end{aligned}
$$

where $h\left(c_{A}, c_{B}, \vartheta\right)$ is the enthalpy and $\left\{k_{i}\right\}_{i=1,2}$ are the reaction rate coefficients modeled with the Arrhenius function.

This example can be found in [13]. Therefore we consider the same setup and control task in order to compare both results with each other. The manipulating input signal is chosen to the cooling power $Q$. The objective is to change the reactor temperature from an initial value $\vartheta(t=0)=373 \mathrm{~K}$ up to $\vartheta\left(t=T^{*}\right)=383 \mathrm{~K}$ as fast as possible. The relative degree is $r=2$ which means that one has to choose two internal state variables.

As basic and variational term $A_{t_{f}}(\tau ; p)$ and $B_{t_{f}}(\tau ; q)$ for the setup function $\Lambda_{t_{f}}$, we use a polynomial ansatz as given in Table 1 . The degree of freedom is $N_{v}=10$. Furthermore, two cases are studied. In the first case, we have input constraints comparable with [13]. In the second case, we additionally constrain the derivative of the manipulating signal in the tmSTP. Table 3 summarizes the variables and constraints of the optimization problem.
In both cases we get an optimal solution for the transition time $T$ and the parameters $q$. The optimal time for a stationary set point transition is $T^{*} \approx 7.44 \mathrm{~min}$ in the first and $T^{*} \approx 8.06 \mathrm{~min}$ for the second case.

Figure 3 shows the resulting state and input trajectory of the original system $\tilde{\Sigma}$. Within the transition horizon $\left[0, T^{*}\right]$ (blue area) the manipulating signal is calculated by the feedback law (19) using the results from the transition problem (16). The application of $\tilde{\alpha}_{x}$ does not change the stability property, so that the plant is still stable. Therefore, an additional feedback controller $\beta$ is not required for nominal stability and is set to zero in this example. We can see in Figure 3, there are state coordinates which have not yet reached the steady state after $t>T^{*}$. Due to the fact that the controlled plant remains stable, we apply (19) over the transition horizon, where $\Lambda_{T^{*}}^{(r)} \equiv 0$. This ensures that the set point remains constant for $t>T^{*}$.

Comparing the results of the first case with those in [13], the state trajectories are similar if the upper input constraint becomes active. The analysis of the transition time depending on the aggressiveness yields a minimal value of $T_{\min } \approx 11 \mathrm{~min}$ for $\delta \rightarrow 1$ for the approach in [13]. The resulting control signal is a nearly bang-bang control ensuring a transition between two steady states. Since we only consider a stationary set point transition, the transition process becomes faster. Adding constraints for the input derivative make the transition time marginally longer, while a bang-bang solution is prevented.

Table 3

Control setup and constraints.

\begin{tabular}{|l|l|l|l|}
\hline & Variable & Constraint & Unit \\
\hline input & $u=Q$ & $\in[-8.5,0]$ & $\mathrm{MJ} / \mathrm{h}$ \\
\hline output & $y=\vartheta$ & $\in[350,400]$ & $\mathrm{K}$ \\
\hline internal states & $c_{A}, c_{B}$ & $\in[0.5,3.5]$ & $\mathrm{kmol} / \mathrm{m}^{3}$ \\
\hline input derivative & $\dot{u}=\dot{Q}$ & $\in[-2,2] \cdot 10^{2}$ & $\mathrm{MJ} / \mathrm{h}^{2}$ \\
\hline transition time & $T$ & $\in \mathbb{R}^{+}$ & $\mathrm{min}$ \\
\hline parameter & $\left\{q_{i}\right\}_{i=1, \ldots, 10}$ & $\in[-1,1] \cdot 10^{4}$ & \\
\hline
\end{tabular}



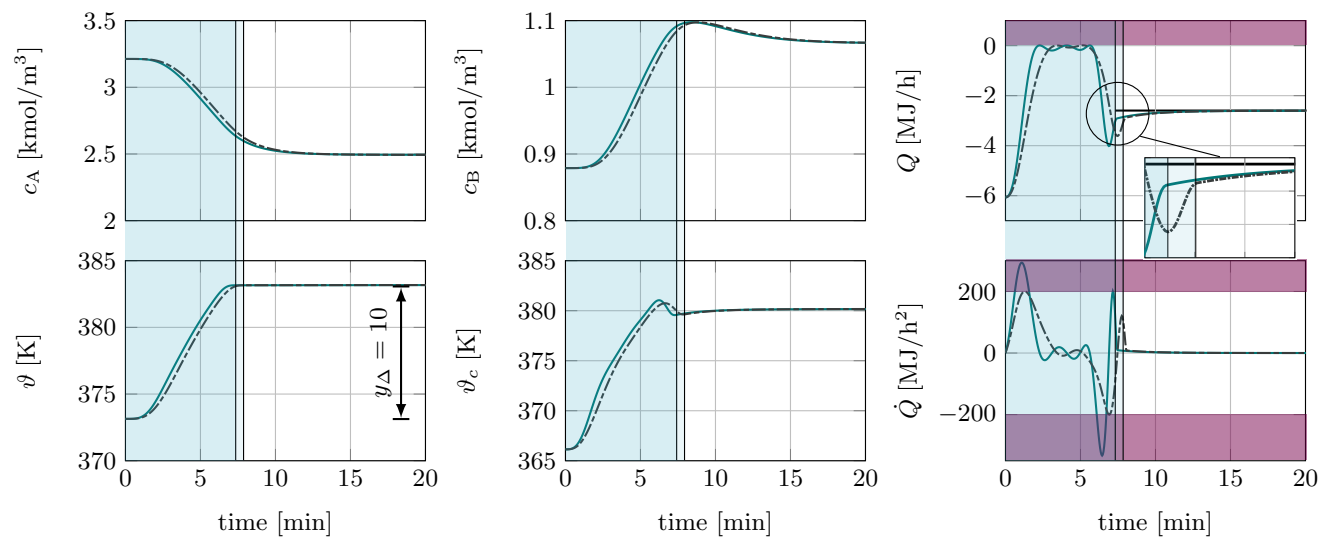

Fig. 3. State and input variables for a output transition of the reactor temperature. The turquois solid curves show the results for first and the dashed grey curves for the second case. The blue area illustrates the transition horizon and the red area the constraints.

\section{Conclusion}

This contribution presents a feedforward control scheme for a time-minimal set point transition in presence of input, state and output constraints. In particular, the use of a novel setup function allows both, simultaneous planning of the output trajectory and calculation of the control signal without violating the initial and terminal conditions. Moreover, we are able to formulate the transition problem as a parameter optimization problem so that the complexity of the time-minimal set point transition problem is reduced to the choice of the type of ansatz function and the number of free paramter. In contrast to the classical approach from Section 3.1, we focus on the stationarity of the final set point and not on the fact that the corresponding state coordinates have reached their final steady state. This way, the transition time can be reduced significantly. Additionally the time derivative of the control signal is considered to restrict the rate, i.e. the first derivative of the manipulating signal, which prevents bang-bang solutions. This is important to minimize wear and to keep the plant in a proper operation. Finally, the novel approach is compared with the classical method. We have demonstrated the performance of the proposed control strategy by applying it to the van de Vusse reactor.

\section{Acknowledgements}

The author Andreas Himmel is also affiliated to the "International Max Planck Research School (IMPRS) for Advanced Methods in Process and Systems Engineering (Magdeburg)."

\section{References}

[1] J.A.E. Andersson, J Gillis, G. Horn, J.B. Rawlings, 7 and M. Diehl. CasADi - A software framework for 72 nonlinear optimization and optimal control. Mathematical Programming Computation, In Press, 2018.

[2] K.J. Astrom and R.M. Murray. Feedback Systems: An Introduction for Scientists and Engineers. Princeton University Press, 1st edition, 2008.

[3] L.T. Biegler. Nonlinear Programming: Concepts, Algorithms, and Applications to Chemical Processes. Series on Optimization. SIAM, 2010.

[4] T. Binder, L. Blank, H.G. Bock, R. Bulirsch, W. Dahmen, M. Diehl, T. Kronseder, W. Marquardt, J.P. Schlöder, and O.v. Stryk. Introduction to Model Based Optimization of Chemical Processes on Moving Horizons. In M. Grötschel, S.O. Krumke, and J. Rambau, editors, Online Optimization of Large Scale Systems: State of the Art, pages 295-340. Springer, 2001.

[5] S. Devasia. Nonlinear minimum-time control with pre- and post-actuation. Automatica, 47(7):1379-1387, 2011.

[6] S. Devasia. Time-optimal control with pre/post actuation for dual-stage systems. IEEE Transactions on Control Systems Technology, 20(2):323-334, 2012.

[7] S. Devasia, D. Chen, and B. Paden. Nonlinear inversionbased output tracking. IEEE Transactions on Automatic Control, 41(7):930-942, 1996.

[8] S. Engell. Feedback control for optimal process operation. Journal of Process Control, 17(3):203-219, 2007.

[9] N. Faiz, S.K. Agrawal, and R.M. Murray. Trajectory planning of differentially flat systems with dynamics and inequalities. Journal of Guidance, Control, and Dynamics, 24(2):219-227, 2001.

[10] T. Faulwasser. Optimization-based solutions to constrained trajectory-tracking and path-following problems. Shaker Verlag, 2013. Number 3 in Contributions in Systems Theory and Automatic Control.

[11] M. Fliess, J. Levine, P. Martin, and P. Rouchon. Flatness and defect of non-linear systems: introductory theory and examples. Int. Journal of Control, 61(6):1327-1361, 1995.

[12] M. Fliess, H. Mounier, P. Rouchon, and J. Rudolph. A distributed parameter approach to the control of a tubular reactor: a multivariable case. In Proceedings of the 37th IEEE Conference on Decision and Control, volume 1, pages 439$442,1998$.

[13] K. Graichen. Feedforward Control Design for FiniteTime Transition Problems of Nonlinear Systems with Input 
and Output Constraints. Berichte aus dem Institut für 6 Systemdynamik, Universität Stuttgart. Shaker, 2006.

[14] K. Graichen and M. Zeitz. Feedforward control design for finite-time transition problems of nonlinear systems with input and output constraints. IEEE Transactions on Automatic Control, 53(5):1273-1278, 2008.

15] L. Grüne and J. Pannek. Nonlinear Model Predictive Control: Theory and Algorithms. Springer Publishing Company, Incorporated, 2013.

16] M. Guay. Real-time dynamic optimization of nonlinear systems: A flatness-based approach. In Proceedings of the 44th IEEE Conference on Decision and Control, pages 58425847, 2005.

[17] A. Isidori. The zero dynamics of a nonlinear system: From the origin to the latest progresses of a long successful story. European Journal of Control, 19(5):369-378, 2013.

[18] Alberto Isidori. Nonlinear Control Systems. Springer-Verlag New York, Inc., 3rd edition, 1995.

[19] B. Käpernick and K. Graichen. Transformation of output constraints in optimal control applied to a double pendulum on a cart. IFAC Proceedings Volumes, 46(23):193-198, 2013. 9th IFAC Symposium on Nonlinear Control Systems.

[20] T. Kleinert, M. Weickgenannt, B. Judat, and V. Hagenmeyer. Cascaded two-degree-of-freedom control of seeded batch 86 crystallisations based on explicit system inversion. Journal of Process Control, 20(1):29-44, 2010.

[21] J. Lévine. Differentially Flat Systems, pages 131-179. Springer Berlin Heidelberg, 2009.

[22] Q. Lin, R. Loxton, and K.L. Teo. The control parameterization method for nonlinear optimal control: A survey. Journal of Industrial \& Management Optimization, 10:275-309, 2014.

[23] Q. Lin, R. Loxton, K.L. Teo, and Y.H. Wu. A new computational method for a class of free terminal time optimal control problems. Pacific Journal of Optimization, $7(1): 63-81,2011$.

[24] C. Liu, Z. Gong, K.L. Teo, R. Loxton, and E. Feng. Biobjective dynamic optimization of a nonlinear time-delay system in microbial batch process. Optimization Letters, 12(6):1249-1264, 2018.

[25] U. Mackenroth. Robust Control Systems: Theory and Case Studies. Springer-Verlag Berlin Heidelberg, 1st edition, 2004.

[26] J. Matschek, T. Bäthge, T. Faulwasser, and R. Findeisen. Nonlinear Predictive Control for Trajectory Tracking and Path Following: An Introduction and Perspective. Birkhäuser, 2018.

[27] D.Q. Mayne, J.B. Rawlings, C.V. Rao, and P.O.M Scokaert. Constrained model predictive control: Stability and optimality. Automatica, 36(6):789-814, 2000.

[28] H. Nijmeijer and A. van der Schaft. Nonlinear Dynamical Control Systems. Springer-Verlag New York, Inc., 1990.

[29] J. Oldenburg and W. Marquardt. Flatness and higher order differential model representations in dynamic optimization. Computers \& Chemical Engineering, 26(3):385-400, 2002.

[30] H. Perez and S. Devasia. Optimal output-transitions for linear systems. Automatica, 39(2):181-192, 2003.

[31] A. Piazzi and A. Visioli. Optimal inversion-based control for the set-point regulation of nonminimum-phase uncertain scalar systems. IEEE Transactions on Automatic Control, 46(10):1654-1659, 2001.

[32] A. Piazzi and A. Visioli. Optimal noncausal set-point regulation of scalar systems. Automatica, 37(1):121-127, 2001.
[33] R. Rothfuss, J. Rudolph, and M. Zeitz. Flatness based control of a nonlinear chemical reactor model. Automatica, 32(10):1433-1439, 1996.

[34] D.E. Seborg, T.F. Edgar, D.A. Mellichamp, and III F.J. Doyle. Process Dynamics and Control. Wiley, 4rd edition, 2016.

[35] S. Skogestad. Control structure design for complete chemical plants. Computers \& Chemical Engineering, 28(1):219-234, 2004.

[36] K. Springer, H. Gattringer, and P. Staufer. On time-optimal trajectory planning for a flexible link robot. Proceedings of the Institution of Mechanical Engineers, Part I: Journal of Systems and Control Engineering, 227(10):752-763, 2013.

[37] M. Treuer, T. Weissbach, and V. Hagenmeyer. Flatness-based feedforward in a two-degree-of-freedom control of a pumped storage power plant. IEEE Transactions on Control Systems Technology, 19(6):1540-1548, 2011.

[38] J.G. van de Vusse. Plug-flow type reactor versus tank reactor. Chemical Engineering Science, 19(12):994-996, 1964.

[39] S. Varigonda, T.T. Georgiou, and P. Daoutidis. Numerical solution of the optimal periodic control problem using differential flatness. IEEE Transactions on Automatic Control, 49(2):271-275, 2004.

[40] A. Visioli. Practical PID Control. Springer-Verlag London, 1st edition, 2006.

[41] P. Wieland, T. Meurer, K. Graichen, and M. Zeitz. Feedforward control design under input constraints for a tubular reactor model. In Proceedings of the 45th IEEE Conference on Decision and Control, pages 3968-3973, 2006.

\section{A Proof of Lemma 12}

Proof: The diagram in Figure 2 illustrates the following. We consider three different coordinate charts $x, z$ and $\tilde{z}$ of state manifold $\mathcal{X}$. As already stated, the coordinate transformation $\Phi: x \mapsto \tilde{z}$ is defined by (2) and the transformation $\Gamma: x \mapsto z$ is given by (10) The first $r$ coordinates of the transformation $\Gamma: x \mapsto \tilde{z}$ are represented by For the first $r$ components applies

$$
\begin{aligned}
\Gamma^{i}(x) & :=\left(\mathrm{L}_{f}^{i-1} h\right)(x)=\left(\mathrm{L}_{T \tilde{f}}^{i-1} h\right)(x) \\
& =T^{i-1}\left(\mathrm{~L}_{\tilde{f}}^{i-1} h\right)(x)=T^{i-1} \Phi^{i}(x)
\end{aligned}
$$

In a more compact form, we can write $\Gamma(x)=\boldsymbol{T} \circ \Phi(x)$ with $\boldsymbol{T}=\operatorname{diag}\left(1, T, \ldots, T^{r-1}, 1, \ldots, 1\right)$. Finally, it follows from Figure 2, that the identity map $i d$ and the rule for inverting a composition of two maps that a change of coordinates $z \mapsto \tilde{z}$ is given by

$$
\begin{aligned}
\Phi \circ \Gamma^{-1} & =\Phi \circ(\boldsymbol{T} \circ \Phi)^{-1}=\Phi \circ\left(\Phi^{-1} \circ \boldsymbol{T}^{-1}\right) \\
& =i d_{\mathbb{R}^{n_{x}}} \circ \boldsymbol{T}^{-1} .
\end{aligned}
$$

94 The diagonal elements of the inverse $\boldsymbol{T}^{-1}=\operatorname{diag}\left(1, T^{-1}\right.$, $\left.95 \ldots, T^{-(r-1)}, 1, \ldots, 1\right)$ are the components in (11). 


\section{B Parameter of $A(\tau ; p)$}

Based on [32], the coefficients for the polynomial term are given by

$$
p_{i}=\frac{(-1)^{i-1}(2 r+1) !}{(i+r) \cdot r !(i-1) !(r+1-i) !} .
$$

The coefficients for the trigonometric series can be computed with Algorithm 1. It should be noted that a high relative degree $r$ can cause an ill-conditioned matrix $A$. At this point further modifications has to be done, e.g. regularisation techniques. The rate of convergence of $\mathrm{Al}-$ gorithm 1 is comparable to determining the solution of a linear equation.

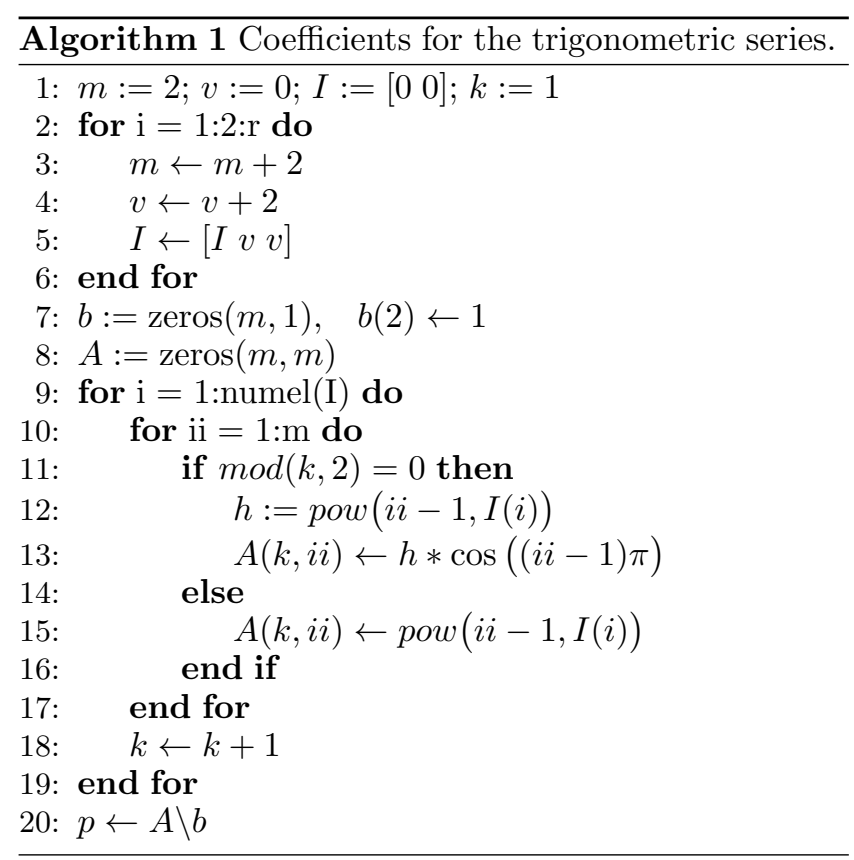

\section{Derive of the System $\Xi$}

Proof: First, we consider equation (8a). It follows from (2a) that $\tilde{z}^{i+1}$ is the time derivative of $\tilde{z}^{i}$. With equation (7a) follows

$$
\begin{array}{r}
\mathrm{L}_{Y} \mu^{i}+\mathrm{L}_{Y} \nu^{i} \psi^{i}\left(\xi^{i} ; \underline{\psi}_{i}, \bar{\psi}_{i}\right) \\
=\mu^{i+1}+\nu^{i+1} \psi^{i+1}\left(\xi^{i+1} ; \underline{\psi}_{i+1}, \bar{\psi}_{i+1}\right)
\end{array}
$$

where $Y$ denotes the vector field to be determined. The first term becomes $\mathrm{L}_{Y} \mu^{i}=\sum_{j=1}^{n_{x}} \frac{\partial \mu^{i}}{\partial \xi^{j}} Y^{j}$. However, $\mu^{i}$ depends only on $\xi^{1}, \ldots, \xi^{i-1}$, the Lie derivative contains only the states up to $\xi^{i-1}$. The second term in (C.1) is splitted into two parts, using the product rule.

$$
\underbrace{\left(\mathrm{L}_{Y} \nu^{i}\right) \psi^{i}\left(\xi^{i} ; \underline{\psi}_{i}, \bar{\psi}_{i}\right)}_{\text {1st part }}+\underbrace{\nu^{i}\left(\mathrm{~L}_{Y} \psi^{i}\left(\xi^{i} ; \underline{\psi}_{i}, \bar{\psi}_{i}\right)\right)}_{\text {2nd part }}
$$

Evaluating the 1st part yields $\psi^{i} \sum_{j=1}^{n_{x}} \frac{\partial \nu^{i}}{\partial \xi^{j}} Y^{j}$.The second term can be splitted into two part represented by $\nu^{i}\left(\sum_{j=1, j \neq i}^{n_{x}} \frac{\partial \psi^{i}}{\partial \xi^{j}} Y^{j}+\frac{\partial \psi^{i}}{\partial \xi^{i}} Y^{i}\right)$. Comparing these expressions with (C.1), one can summarize

$$
\begin{aligned}
\mu^{i+1} & =\sum_{j=1}^{n_{x}}\left[\left(\frac{\partial \mu^{i}}{\partial \xi^{j}}+\frac{\partial \nu^{i}}{\partial \xi^{j}}\right) Y^{j}\right]+\nu^{i} \sum_{j=1, j \neq i}^{n_{x}} \frac{\partial \psi^{i}}{\partial \xi^{j}} Y^{j} \\
\nu^{i+1} & =\nu^{i} \frac{\partial \psi^{i}\left(\xi^{i} ; \underline{\psi}_{i}, \bar{\psi}_{i}\right)}{\partial \xi^{i}} \\
Y^{i} & =\psi^{i+1}\left(\xi^{i+1} ; \underline{\psi}_{i+1}, \bar{\psi}_{i+1}\right) .
\end{aligned}
$$

Considering the last equation, the individual saturation functions define the components of the vector field for the first $r-1$ coordinates. Next, we come to equation (8b). Due to the diffeomorphism $\Psi$ the component of the vector field for the $r$-th coordinate consists of a saturation function, too. Instead of $\xi^{r+1}$, one introduce $v$ and call it new input. Finally, we consider equation (8c). Due to $(7 \mathrm{~b})$, the internal states are determined by $(2 \mathrm{~b})$ with the associated vector field components (3). If Assumption 2 holds, one can choose internal states whose dynamic are not influenced by the input $u$. Additional the coordinate transformation from $\xi \mapsto x$ are included, see the diagram in Figure 2,

Another result of this proof is an iteration rule to compute the transformation law $\Psi$. Consider the case $i=1$, where $\mu^{1}:=0$ and $\nu^{1}:=1$ are independent on $\xi$. The term $\mu^{2}$ becomes zero, due to the partial derivatives. The term $\nu^{2}=\frac{\partial \psi^{1}}{\partial \xi^{1}}$ depends on $\xi^{1}$. 